\title{
Uma Análise dos Padrões de Tatuagens Associados à Criminalidade do Estado da Bahia com Auxílio da Teoria de Redes
}

\section{Hernane Borges de Barros Pereira ${ }^{1,3}$, Antônio José Assunção Cordeiro ${ }^{1,2}$, Carlos César Ribeiro Santos ${ }^{1}$, Alden José Lázaro da Silva ${ }^{1}$}

${ }^{1}$ Programa de Modelagem Computacional, SENAI Cimatec, Salvador, Bahia, Brasil

${ }^{2}$ Centro Universitário Estácio da Bahia - Estácio FIB - Salvador, BA - Brazil

${ }^{3}$ Universidade do Estado da Bahia (UNEB), Salvador, Bahia, Brasil

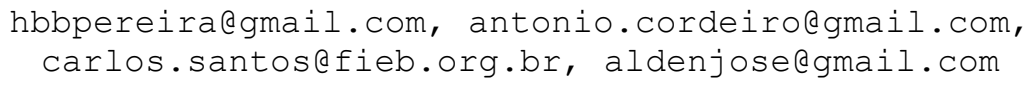

\begin{abstract}
This paper presents an analysis of tattoos patterns associated with occurrences of crimes recorded by the Department of Public Safety State of Bahia, using the Networks theory. Networks built consider the relationship between criminals, crimes and tattoos. Networks present Small World phenomenon. The results show relevant data for the understanding of crime patterns associated with tattoos and may eventually support the public security authorities in decision
\end{abstract}

Resumo. Este artigo apresenta uma análise de padrões de Tatuagens associadas a ocorrências de crimes registrados pela Secretaria de Segurança Pública do Estado da Bahia utilizando a teoria de Redes Sociais e Complexas. As redes construidas consideram as relações existentes entre criminosos, crimes e tatuagens. Os resultados apresentados apontam redes com característica topológica Small World. Os resultados encontrados apontam dados relevantes para a compreensão dos padrões de criminalidade associados a tatuagens, podendo apoiar futuramente as autoridades de segurança pública na tomada de decisão.

\section{Introdução}

O estudo da segurança pública no Brasil vem crescendo dentro do contexto acadêmico. Percebe-se uma aproximação das instituições de segurança no país com a comunidade científica. Estados e municípios buscam por soluções otimizadas para a realidade da violência e criminalidade em que estão inseridas. Tais demandas podem ser respondidas a partir de diversos estudos e métodos, dentre os quais se destaca a Teoria de Redes.

Uma rede social é uma estrutura capaz de representar a ligação entre indivíduos, grupos de indivíduos, organizações, etc. (e.g. crimes, tatuagens, adesão a facções criminosas). É uma das formas de representação dos relacionamentos efetivos ou profissionais de indivíduos entre si, ou entre seus agrupamentos de interesse mútuos (PEREIRA, 2013).

Pode-se afirmar que a estrutura da segurança pública de um estado é formada por diversos atores e relações que variam de acordo com entidades, áreas ou sub-áreas a que estão diretamente ou não relacionados. Policiais, órgãos, crimes cometidos, locais de crimes, criminosos, tatuagens de criminosos, entre outras características, podem formar 
uma extensa redes de relações. Notamos algumas redes sociais nesse âmbito.

Vale ressaltar que esforços têm sido feitos pelo Estado da Bahia na aproximação deste com a comunidade científica. Durante alguns anos da década passada, inseridos no Programa de Políticas Públicas, a Fundação de Amparo à Pesquisa do Estado da Bahia (FAPESB) lançou os Editais Temáticos que tinham como foco a prevenção e o combate a violência e criminalidade, além de estimular a atividade de pesquisa em segurança pública nas universidades do Estado da Bahia.

Em 2014, o programa de Pós-Graduação em Modelagem Computacional e Tecnologia Industrial da Faculdade de Tecnologia Senai Cimatec-BA, obteve da Polícia Militar do Estado uma base de dados que contém uma pesquisa das relações existentes entre criminosos, tatuagens e crimes e um a cartilha com a síntese da proposta de análise e atuação (SILVA, 2014). Percebeu-se que importantes respostas sobre o comportamento e características dessas relações poderiam ser obtidas do o uso da teoria de redes. A proposta de análise considera as relações existentes entre tatuagens e crimes, criminosos e tatuagem e crimes e criminosos.

Assim, a compreensão dos padrões de interação entre os atores analisados neste trabalho destaca o comportamento da criminalidade, sobre tudo, a questão central deste estudo: Quais informações estratégicas, para a segurança pública do Estado da Bahia, podem ser obtidas com o uso da análise das redes sociais e complexas provenientes de uma base de dados de Tatuagens em Criminosos?

O objetivo principal desse trabalho é modelar as relações entre criminosos e tatuagens, crimes e tatuagens e crimes e criminosos a partir da base de dados de Tatuagens da Polícia Militar da Bahia, utilizando técnicas e parâmetros da análise de redes sociais e da teoria de redes complexas.

A importância dessa pesquisa relaciona-se com uma sistematização de um método capaz de auxiliar pesquisadores, setores de inteligência da segurança pública e, principalmente, policiais, a compreender e explicar como se dão relações de interesse entre criminosos, tatuagens e crimes, contribuindo sensivelmente para futuras políticas públicas de segurança mais assertivas.

\section{Fundamentação Teórica}

Este artigo fundamenta-se em três importantes áreas teóricas: a teoria dos grafos, a análise de redes sociais e a teoria das redes complexas. Tais teorias têm sido aplicadas na resolução dos mais variados problemas, para diferentes áreas do conhecimento visto que têm obtido relevantes resultados para a tomada de decisão em empresas, pesquisas biológicas, tecnologia da informação, organizações de diferentes naturezas, universidades, entre outros.

O fundamento matemático dessa aplicação advém da teoria dos grafos, ramo da matemática que estuda as relações entre os objetos de um determinado conjunto. Para tal são empregadas estruturas chamadas de grafos, representado por $G=(V, E)$, onde $V$ é um conjunto não vazio de objetos denominados vértices e finito e $E$ é um conjunto de pares não ordenados de $V$, chamado de arestas.

Destaca-se ainda que uma rede é considerada complexa quando seu tamanho é suficientemente grande de modo que algumas propriedades emergem. Por sua vez, uma rede é considerada social quando a mesma lida com relações entre pessoas. As 
pesquisas, antes focadas nas pequenas redes e nas propriedades de vértices individuais ou arestas, passaram a considerar propriedades estatísticas em larga escala (Metz et al., 2007).

Uma rede complexa pode apresentar topologias importantes, dentre as quais podemos destacar as seguintes: (1) Redes de Grafos Aleatórios (Erdös; Rényi, 1960); (2) Redes de Mundo Pequeno (Small World) (Watts;Strogatz,1998); c) Redes Livres de Escala (Scale Free) (Barabasi;Albert:1999).

Nas redes de grafos aleatórios, cada um dos vértices tem a mesma probabilidade de receber novas ligações. Nestas redes, a distribuição de conectividade decai exponencialmente. As redes mundo-pequeno (Small World) são caracterizadas por possuírem um alto grau de aglomeralção e caminho mínimo médio similar ao de uma rede aleatória. A rede livre de escala possui uma distribuição de conectividade que segue uma lei de potência. Ou seja, vértices com muitas ligações possuem uma probabilidade maior de receber novas ligações.

As redes aqui apresentadas são redes de 1-modos. Com elas, analisamos o comportamento dos criminosos no que se refere aos crimes cometidos e tatuagens utilizadas por eles, bem como a relação existente entre tatuagens que podem representar determinados tipos de crimes.

\section{Método}

Conforme já citado, a proposição deste artigo é construir e analisar redes de criminosos a partir de um banco de dados de tatuagens identificadas em criminosos oriundo da Polícia Militar do Estado da Bahia. A construção das redes iniciou-se com a delimitação de relevantes informações:

- Catálogo com mil criminosos: presos ou em processo. Identificados sequencialmente;

- Crimes Cometidos (tipos): sete tipos de crimes analisados que foram furto, roubo, associação ao crime, narcotráfico, homicídio, latrocínio e associação ao crime;

- Tatuagens: total de 60 tatuagens diferentes catalogadas na base de dados;

- Locais: denominados de Áreas Integradas de Segurança Pública (AISP), são os locais da região soteropolitana em que os crimes foram cometidos.

Com a base de dados e informações devidamente desnormalizadas, considerando-se ainda os fundamentos teóricos, construímos as redes sociais de indivíduos com base nos critérios Crimes-Tatuagens e nos critérios Crimes-Tatuagens-Localidade através da utilização dos softwares Pajek e Gephi. A próxima seção, apresentaremos a análise das redes de tatuagens.

\section{Resultados e Discussão}

A base de dados utilizada é restrita ao município de Salvador, denotando uma lacuna para posterior análise de dados outros municípios do estado e do País.

Em um primeiro momento, esta pesquisa realizou o tratamento dos dados de modo a normalizá-los, viabilizando assim a concepção das redes. Essas foram construídas vinculando os indivíduos através da ocorrência de mesma tatuagem e crime praticado. Desta forma, criminosos que possuíssem a mesma tatuagem estariam conectados entre si na rede. Porém, esta abordagem resultou em redes com uma densidade muito alta (mais de 29\%) o que inviabiliza parte da análise, uma vez que as redes ficam altamente 
conectadas.

Por esse motivo, o foco da pesquisa configurou-se em construir redes com mais de um critério para estabelecer de vínculos entre os indivíduos. Assim, foram montadas redes de criminosos com vínculos definidos pela coexistência de pelo menos dois fatores em comum, a saber: mesma tatuagem e mesmo crime cometido.

Tabela 1. Características das Redes de Tatuagens

\begin{tabular}{|cccccccc|}
\hline Redes & Vértices & Arestas & Grau M. & Densidade & C. $\mathbf{M}$. M. & Diâmetro & Cws \\
& $|\mathbf{v}|$ & $|\mathbf{E}|$ & $<\mathbf{k}>$ & $\Delta$ & $\mathbf{L}$ & & \\
R1-CT & 763 & 141.181 & 370,068 & 0,486 & 1,514 & 2 & 0,844 \\
A1-CT & 763 & 141.287 & 370,346 & 0,486 & 1,513 & 2 & 0,486 \\
R2-CLT & 666 & 78.975 & 237,162 & 0,357 & 1,653 & 3 & 0,843 \\
A2-CLT & 666 & 79.039 & 237,354 & 0,356 & 1,643 & 2 & 0,356 \\
\hline
\end{tabular}

A Tabela 1 mostra os dados das redes criadas e das redes aleatórias geradas a partir da quantidade de seus vértices e graus médios. A primeira rede vincula os criminosos entre si que tenham cometido os mesmos crimes e que tenham as mesmas tatuagens. As informações dessa rede aparecem na primeira linha da tabela, com a referência R1-CT. A segunda linha da tabela contém as informações da rede aleatória (A1-CT) criada com base no número de vértices e grau médio da R1-CT.

A rede R1-CT (Figura 1), analisada através do maior componente, teve sua topologia identificada como Small World, visto que a distância entre qualquer par de vértices na rede é pequena. Além disso, seu coeficiente de aglomeração é maior do que o coeficiente de aglomeração da rede A1-CT, e seu caminho mínimo médio foi similar ao da mesma rede A1-CT. A caracterização da Rede R1-CT ilustra a ocorrência de dois eventos distintos, associando uma aresta entre indivíduos que possuem uma relação pelos dois eventos: (1) cometeram o mesmo crime; (2) possuem a mesma tatuagem. Esta relação possibilita a um gestor de segurança pública a identificação de grupos de tatuagens que se aglomeram por alguma prática dos crimes aqui analisados. Neste sentido, o gestor poderá perceber os padrões que explicam como os criminosos estão utilizando tatuagens para sua identificação criminosa. Destaca-se ainda a possibilidade de compreender a influência gerada pela distância entre criminosos que praticaram crimes distintos, e, até mesmo a distância entre indivíduos que cometeram crimes considerados mais brandos para crimes considerados mais graves, uma vez que muitos criminosos acabam cometendo vários tipos de crimes distintos.

A Rede R2-CLT (Figura 2) vincula os criminosos entre si, que tenham as mesmas tatuagens, cometido os mesmos crimes e atuado na mesma localidade. Essa rede é também Small World. A rede aleatória foi criada com base no número de vértices e grau médio da R2-CLT. Destaca-se que essa análise também utilizou a maior componente da rede R2-CLT. A relevância das informações geradas na Rede R2-CLT consiste na possibilidade do gestor de segurança pública poder identificar localidades onde os criminosos com padrões de associação de tatuagens se reúnem para realizar práticas criminosas, possibilitando o desenvolvimento de ações e políticas de policiamento ostensivo localizado, investigações de facções criminosas e a distância entre estes eventos (prática de Crimes, tatuagens localidades). 


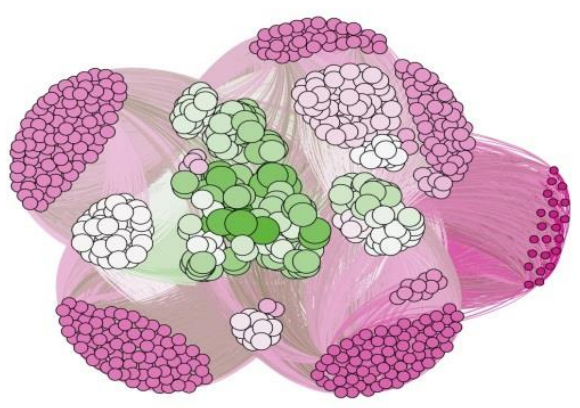

Figura 1. Rede R1-CT

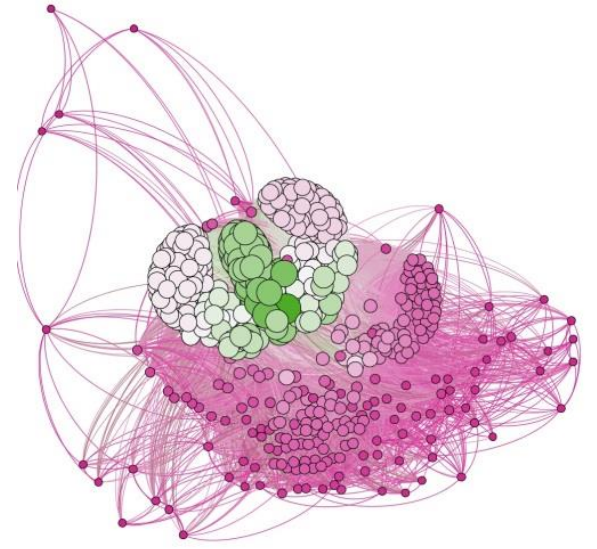

Figura 2. Rede R2-CLT

Considerando nosso modelo de análise em que um indivíduo pode ser ligado a outro, caso o mesmo tenha cometido o mesmo crime e que possua a mesma tatuagem, podemos dizer que um par de vértices é alcançável caso essa relação seja verdadeira, aumentando a chance de ser verdadeira a afirmação de que uma devida tatuagem represente um determinado crime. Estatisticamente, podemos afirmar que as tatuagens na Tabela 2 representam um crime específico, pois a tatuagem foi encontrada em $100 \%$ dos indivíduos que cometeram determinado crime.

\begin{tabular}{c|c|c|c}
\hline Tatuagem & Crime & Qtde & \% \\
\hline $\begin{array}{c}\text { Paz Justiça } \\
\text { Liberdade }\end{array}$ & $\begin{array}{c}\text { Associação ao } \\
\text { Crime }\end{array}$ & 241 & $100 \%$ \\
\hline Bruxo & Furto & 110 & $100 \%$ \\
\hline 121 & Homicídio & 217 & $100 \%$ \\
\hline Coringa & Latrocínio & 295 & $100 \%$ \\
\hline Carpa & Narcotráfico & 274 & $100 \%$ \\
\hline Palhaço & Roubo & 262 & $100 \%$ \\
\hline
\end{tabular}

Tabela 2. Vínculos Estatísticos de 100\%

\begin{tabular}{c|c|c|c}
\hline Tatuagem & Crime & Qtde & \% \\
\hline Coringa & Roubo & 168 & $64 \%$ \\
\hline Palhaço & Latrocínio & 169 & $57 \%$ \\
\hline Caveira & $\begin{array}{c}\text { Associação } \\
\text { ao Crime }\end{array}$ & 130 & $54 \%$ \\
\hline Coringa & Furto & 57 & $52 \%$ \\
\hline
\end{tabular}

Tabela 3. Vínculos Relevantes de Menos de 100\%

Estatisticamente, pode-se afirmar que há um grau elevado de possibilidade das tatuagens abaixo representarem um crime, pois foi possível observá-las em mais de $50 \%$ das ocorrências analisadas (Tabela 3):

Com relação às demais tatuagens, não temos condições de afirmar que as mesmas estão associadas a um determinado tipo de crime, pois não foi constatado em mais de $50 \%$ das ocorrências (Tabela 4):

\begin{tabular}{c|c|c|c}
\hline Tatuagem & Crime & Qtde & \% \\
\hline 157 & Latrocínio & 146 & $49 \%$ \\
\hline Palhaço & Furto & 45 & $41 \%$ \\
\hline Carpa & Associação ao Crime & 91 & $38 \%$ \\
\hline 157 & Roubo & 98 & $37 \%$ \\
\hline Arma Fogo & Latrocínio & 106 & $36 \%$ \\
\hline Arma Fogo & Roubo & 90 & $34 \%$ \\
\hline Coringa & Homicídio & 74 & $34 \%$ \\
\hline Caveira & Narcotráfico & 93 & $34 \%$ \\
\hline 121 & Furto & 37 & $34 \%$ \\
\hline Paz Justiça Liberdade & Narcotráfico & 92 & $34 \%$ \\
\hline Palhaço & Homicídio & 69 & $32 \%$ \\
\hline
\end{tabular}




\begin{tabular}{c|c|c|c}
\hline Jesus & Roubo & 79 & $30 \%$ \\
\hline PCC & Latrocínio & 88 & $30 \%$ \\
\hline
\end{tabular}

Tabela 4. Vínculos de Baixa Relevância

\section{Considerações Finais}

O modelo e a análise propostos permite-nos responder à questão de pesquisa: Quais informações estratégicas, para a segurança pública do Estado da Bahia, podem ser obtidas com o uso da análise das redes sociais e complexas provenientes de uma base de dados de Tatuagens em Criminosos?

Por um lado, observamos que é factível, por inspeção visual e por análise de agrupamento, identificar os membros de facções criminosas através de tatuagens. Por outro lado, a análise de influência a partir dos crimes cometidos e das tatuagens dos criminosos evidencia a distância entre indivíduos que cometeram crimes considerados mais brandos e crimes considerados mais graves. Esse tipo de constatação permite à segurança pública antecipar-se a situações criminosas, uma vez que muitos criminosos acabam cometendo vários tipos de crimes distintos ao longo do tempo.

A combinação das medidas de redes apresentadas nesse artigo, a partir da determinação dos atributos dos atores, fornecem diversos subsídios para a análise da estrutura dos arranjos e as respectivas relações entre os criminosos em questão. Os resultados encontrados também inferem novos questionamentos à pesquisa aqui apresentada.

\section{Referências}

Barabási, A. L.; Albert, R. (1999) "Emergence of Scaling in Random Networks". Science, v. 286, p. 509-512.

Erdös, P.; Rényi, A. (1960) "On the evolution of random graphs". Publications of the Mathematical Institute of the Hungarian Academy of Sciences, n. 5, p. 17-61.

Metz, J. et al. (2007) "Redes Complexas: conceitos e aplicações", São Carlos: [s.n.]..

Plano Estadual da Segurança Pública - PLANESP: Governo da Bahia - 2012 a 2015 (2011). Secretaria da Segurança Pública. - Salvador: Secretaria da Segurança Pública.

Pereira, H. B. B. (2013) "Redes Sociais e Complexas: Aplicações em Difusão do Conhecimento". Academia de Ciências da Bahia: Memória, v. 3, p. 39-47.

Silva, Alden L. (2011) "Tatuagem: desvendando segredos ", Salvador: Magic Gráfica, $74 \mathrm{p}$.

Watts, D. J. \& Strogatz, S. H. (1998) "Collective dynamics of 'small-world' networks", Nature, v. 393, n. 4, p. 440-442. 